(c) American Dairy Science Association, 2005.

\title{
The Effect of Synchronization on Genetic Parameters of Reproductive Traits in Dairy Cattle
}

\author{
R. C. Goodling, Jr., ${ }^{\star}$ G. E. Shook, K. A. Weigel, and N. R. Zwald \\ Department of Dairy Science, University of Wisconsin, Madison 53706
}

\section{ABSTRACT}

Genetic evaluation and selection is one strategy for improving female reproductive performance. Many producers use synchronization of ovulation or estrus to manage reproduction. The objective of this study was to examine the effects of reproductive synchronization on genetic parameter estimates of days to first breeding (DFB), days open (DO), and pregnancy rate at $120 \mathrm{~d}$ postpartum (PR120). Data were collected from 64 producers participating in an artificial insemination progeny testing program and using Dairy Comp 305 herd management software to record reproductive treatments and events. Data included 18,359 records for DFB and 16,379 records for DO and PR120. Synchronization was classified by breeding codes at time of insemination. The traits DFB and DO were analyzed using a linear model with age at calving, herd-year-season, and parity as fixed effects and sire and residual as random effects. For PR120, a threshold sire model was used with fixed effects as in the DFB and DO models. Three models were applied to the complete data sets of all traits; a base model with no synchronization effect, an expanded model with a fixed synchronization effect, and an interaction model with a random sire by herd management interaction. Herd management categories were based on an individual herd's use of synchronization protocols. Also, data subsets were analyzed separately based on cow synchronization treatment and herd management categories. Synchronized records for DFB had on average $40 \%$ higher sire variance and $60 \%$ lower residual variance than nonsynchronized records. Heritability for DFB ranged from 0.01 to 0.09 . Sire variance was $40 \%$ lower for DO and $25 \%$ lower for PR120 in first synchronized records than either latersynchronized or nonsynchronized records. Residual variances for DO varied by $3 \%$ among cow treatment categories and $14 \%$ for herd management categories. Heritabilities ranged from 0.03 to 0.07 for DO and 0.10

Received October 31, 2004

Accepted February 27, 2005.

Corresponding author: R. C. Goodling, Jr.; e-mail: rcg133@psu.edu.

*Current address: Penn State Cooperative Extension, Lebanon County, 2120 Cornwall Road, Suite 1, Lebanon, PA 17042. to 0.26 for PR120. Including a fixed effect for synchronization in the DO model reduced sire variance by $33 \%$ and residual variance by $10 \%$. Sire by herd management interactions were less than $2 \%$ of the total variance for all traits. Accounting for synchronization, especially for DFB, may improve accuracy of genetic parameter estimates and animal evaluations.

(Key words: reproductive synchronization, heritability, days to first breeding, days open)

Abbreviation key: DFB = days to first breeding, DO = days open, PR120 = pregnancy rate at $120 \mathrm{~d}$.

\section{INTRODUCTION}

In recent years, the decline in dairy cattle fertility has become a concern. This decline causes substantial losses in the dairy industry (Royal et al., 2002), and raises the economic importance of reproductive function (Van Arendonk et al., 1989). Fertility traits have been included in sire selection indices for several years in Scandinavian countries but only recently in the United States. Choosing appropriate measures for selection is important for achieving genetic progress in fertility traits.

A measure used widely in Europe is days to first breeding (DFB), which has been advocated by several authors (Van Arendonk et al., 1989; Hageman et al., 1991; Royal et al., 2002). This trait can be defined as the interval from calving to the first breeding. Many physiological events occur during this interval, and the trait is an indicator of several aspects of reproductive function. Extended DFB could be due to poor endocrine function, metritis, or subdued estrus expression. Reported DFB means and standard deviations have increased in recent years (Van Arendonk et al., 1989; Hageman et al., 1991; Veerkamp et al., 2001). The increase in DFB could be a reflection of producers delaying breeding due to increased production, but it is also likely that reproductive health of dairy cattle has been declining. Although DFB indicates many of the early factors involved with a cow's recovery from parturition, other traits can provide additional information.

Days open (DO), a trait representing the number of days from calving to conception, is a widely used mea- 
sure of reproduction, especially in the United States. One can view DO as the sum of 2 intervals, DFB and service period (the interval from first breeding to conception). Limiting factors with respect to DO are low accuracy in determining if conception has occurred, especially in the absence of a veterinary pregnancy check, and potential loss of pregnancy after pregnancy diagnosis. A cow may not be seen in estrus after breeding, suggesting conception, when in fact it has not conceived. Ultrasonography to determine pregnancy is more accurate than the common practice of rectal palpation, but is more expensive and time consuming (Fricke, 2002b). With more accurate determination of conception via palpation or ultrasonography, the usefulness of DO in genetic improvement programs may improve. Another shortcoming of DO is that it is truly only defined for cows that become pregnant. In practice, nonpregnant cows are often assigned an arbitrary, high value. The reproductive decline observed in DFB over time has also been observed in DO (Marti and Funk, 1994; Abdallah and McDaniel, 2000).

Because DO, like DFB, has limited recording accuracy in field data, a trait measured at an intermediate time may be advantageous. Pregnancy rate is widely used in herd reproductive management, being measured at particular breedings or time intervals. Pregnancy rate for a time interval is the number of cows that conceive divided by the number of cows eligible for breeding during that interval. For purposes of this study, we chose to investigate pregnancy rate at an interval of $120 \mathrm{~d}$ (PR120). This definition shows that PR120 is dependent on conception rate and service rate of a group of cows. To create an individual trait, PR120 was a binomial trait defined as a "success" (pregnancy or abortion by $120 \mathrm{~d}$ ) or a "failure" (no confirmation by $120 \mathrm{~d}$ ).

Animal physiologists have developed hormonal protocols to aid producers in reproductive management. These schemes induce estrus or synchronize animals to ovulate within a particular timeframe, and some even eliminate the need for estrus detection (Jobst et al., 2000). Pursley et al. (1995, 1997) reported that a sequence of $\mathrm{GnRH}$ and $\mathrm{PGF}_{2 \alpha}$ injections, followed by insemination after a particular interval, was as effective as detection of estrus for lactating cows. Other treatments include, but are not limited to, progesterone or prostaglandin injections, Selectsynch, Heatsynch, and Cosynch (Cartmill et al., 2001; Fricke, 2002a). Presynchronization or resynchronization protocols also exist that include injections before or after a standard protocol (Fricke, 2002a). Herd practices and goals determine which, if any, protocol(s) will be implemented.

The objective of this study was to examine the influence of reproductive synchronization on genetic param- eter estimates for DFB, DO, and PR120. We use the term reproductive synchronization to refer to breeding resulting from an attempt to synchronize ovulation. The use of such protocols interferes with the natural reproductive expression of a cow, potentially causing genetic and environmental variation to be modified compared with conventional reproductive management. Accounting for reproductive management practices and synchronization treatments could improve the accuracy of genetic evaluations for reproductive traits.

\section{MATERIALS AND METHODS}

\section{Data}

Data from 64 US dairy herds in an AI progeny testing program were available. All herds used Dairy Comp 305 herd management software (Valley Agricultural Software, Tulare, CA). Individual reproductive events were extracted, including date of breeding, breeding codes (standing heat, timed breeding, etc.), and bred results (pregnant, aborted, open, etc.). Breeding codes were inconsistent across herds and were standardized manually. Herds with less than 50\% sire identification or less than $75 \%$ of inseminations with breeding codes were excluded.

Further requirements included: a recorded fresh event for the lactation; parity $\leq 6$; year of calving between 1999 and 2002; and $\geq 1$ recorded breeding. Only daughters of sires with at least 4 progeny were retained. Incomplete lactations were included if at least one breeding had a corresponding outcome. The short time frame of data collection limited the number of lactations per cow. Therefore, only the earliest record was included for cows with multiple complete lactations. These restrictions were applied to all 3 traits. Further restrictions included the removal of 216 observations with DFB $<37 \mathrm{~d}$ or $>300 \mathrm{~d}$. The data for DO and PR120 had additional limitations based on the range of $\mathrm{DO}$ and the availability of pregnancy confirmation; 994 records with DO > $500 \mathrm{~d}$ or $<37 \mathrm{~d}$ were excluded, and 966 records with no pregnancy confirmation were excluded.

For PR120, a record was classified as a "success" if a breeding before $120 \mathrm{~d}$ postpartum was reported as pregnant or aborted. A record was classified as a "failure" if no breedings before $120 \mathrm{~d}$ resulted in a reported pregnancy or abortion. The 120-d interval reflects an economically desirable calving interval and (with an average success rate of $40 \%$ ) it leads to few herd-yearseason categories with all successes or all failures.

\section{Cow Treatment Approach}

Two approaches, referred to as cow treatment and herd management, were used to classify the data based 
Table 1. Average percentage of synchronized first inseminations and average within-herd standard deviation of days to first breeding (DFB) by herd management category. The ranges of values among herds within each category are in parentheses.

\begin{tabular}{lllll}
\hline $\begin{array}{l}\text { Herd } \\
\text { management } \\
\text { group }\end{array}$ & $\begin{array}{l}\text { No. of } \\
\text { herds }\end{array}$ & $\begin{array}{l}\text { Percentage of } \\
\text { records with } \\
\text { unreported type } \\
\text { of breeding, \% }\end{array}$ & $\begin{array}{l}\text { Percentage of } \\
\text { synchronized first } \\
\text { inseminations, }{ }^{1} \%\end{array}$ & $\begin{array}{l}\text { Within-herd } \\
\text { standard deviation } \\
\text { of DFB, d }\end{array}$ \\
\hline Observed heat & 17 & $7.9(0.0-44.4)$ & $19.1(0.0-37.0)$ & $30.1(21.7-52.7)$ \\
Timed breeding & 11 & $8.9(0.0-70.0)$ & $81.4(66.2-99.5)$ & $13.7(5.1-20.1)$ \\
Mixed & 36 & $7.7(0.0-56.1)$ & $41.5(0.0-100.0)$ & $21.7(9.5-46.0)$ \\
All herds & 64 & $8.0(0.0-70.0)$ & $42.5(0.0-100.0)$ & $22.5(5.1-52.7)$ \\
\hline
\end{tabular}

${ }^{1}$ Percentage adjusted to exclude inseminations with unreported type of breeding.

on synchronization. In the cow treatment approach, synchronization status was determined by the standardized breeding code associated with each individual breeding. For example, a code of "S" signified a breeding resulting from standing heat, whereas "T" signified a timed insemination breeding. Codes such as timed breeding, Ovsynch, etc., classified a breeding as synchronized. Codes such as standing heat, vet palpation, activity, etc., were classified as nonsynchronized. Noncoded breedings were also assumed to be nonsynchronized. For DFB, records were coded as synchronized first breeding or nonsynchronized first breeding. For DO and PR120, records were classified as synchronized first breeding, synchronized later breeding, or nonsynchronized breeding. One limitation to this approach, because of the data structure, is the inability to determine if a breeding coded as standing heat may have been aided by a protocol.

\section{Herd Management Approach}

Herd management classification was separate from, but dependent on, cow treatment, and classification was the same for all records within a given herd. Preliminary classification of herds was based on scatter plots for first and second breeding dates by DIM. Timed breeding herds were characterized by very narrow distribution of DIM at first breeding and a high percentage of synchronized breedings, whereas observed heat herds were characterized by few synchronized breedings and broad range of days at first breeding. Mixed herds had scatter plots that did not conform to either extreme.

After preliminary graphing, 2 parameters were chosen to classify herds: percentage of synchronized first breedings, and within-herd standard deviation of DFB. Percentage of synchronized first breedings was based on the breeding codes associated with each breeding record, as described in the cow treatment approach, and excluded records with no type of breeding reported. The mean over all herds was $42.5 \%$ synchronized first breedings, and the mean within herd standard deviation of DFB was $22.5 \mathrm{~d}$. Herds below $37 \%$ synchronized first inseminations and standard deviation for DFB > $21.5 \mathrm{~d}$ were classified as observed heat herds. Herds above $66 \%$ synchronization and standard deviation for $\mathrm{DFB}<20 \mathrm{~d}$ were classified as timed breeding herds. The mixed management classification was assigned to all other herds. The herd management categories were used to create separate data sets for analysis. The means and ranges for percentage of records with unreported type of breeding, percentage of synchronized first inseminations, and within herd standard deviation of DFB for the herd management groups are in Table 1.

\section{Statistical Models}

Three models were applied to estimate genetic and residual variances: a base model, an expanded model, and an interaction model. The base model was a linear model that included fixed effects for herd-year-season, parity, and age at calving (in months). Parity classes were first, second, and third or greater. Four seasons based on date of calving were defined in 3-mo intervals starting with January. Random effects were sire and residual for all models. The expanded model was comprised of the base model with an additional fixed effect for cow treatment category. The interaction model was comprised of the expanded model with an additional fixed effect for herd management category and a random sire by herd management interaction. Numbers of herds, animals with records, sires, calving age levels, and herd-year-season levels are shown in Table 2.

Initial variance estimates were calculated with REMLF90 (I. Misztal, Animal and Dairy Science Department, University of Georgia, Athens). Results from this analysis were used as starting values for analysis by GIBBS2F90 (I. Misztal), and uninformative priors were assumed. The complete data set was analyzed with the base, expanded, and interaction models for both sire and animal models. Animal model results are not reported because they were similar to sire model 
Table 2. Numbers of herds, animals with records, sires, calving age levels, and herd-year-season levels for the 3 reproductive traits ${ }^{1}$ by cow treatment subset, herd management subset, and complete data. ${ }^{2}$

\begin{tabular}{|c|c|c|c|c|c|c|}
\hline Data group & Subset & Herds & Animals & Sires & $\begin{array}{l}\text { Calving } \\
\text { age } \\
\text { levels }\end{array}$ & $\begin{array}{l}\text { Herd-year- } \\
\text { season } \\
\text { levels }\end{array}$ \\
\hline \multicolumn{7}{|l|}{ DFB } \\
\hline \multirow[t]{2}{*}{ Cow synchronization treatment } & First & 61 & 6435 & 1028 & 80 & 411 \\
\hline & None & 64 & 11,924 & 1159 & 86 & 502 \\
\hline \multirow[t]{3}{*}{ Herd management group } & Observed heat & 17 & 3465 & 675 & 81 & 129 \\
\hline & Timed breeding & 11 & 4129 & 619 & 80 & 90 \\
\hline & Mixed & 36 & 10,765 & 1065 & 84 & 306 \\
\hline Complete data & & 64 & 18,359 & 1174 & 87 & 525 \\
\hline \multicolumn{7}{|l|}{ DO and PR120 } \\
\hline \multirow[t]{3}{*}{ Cow synchronization treatment } & First & 60 & 5540 & 977 & 79 & 275 \\
\hline & Later & 61 & 2598 & 751 & 73 & 193 \\
\hline & None & 64 & 8241 & 1105 & 85 & 360 \\
\hline \multirow[t]{3}{*}{ Herd management group } & Observed heat & 17 & 3013 & 649 & 81 & 114 \\
\hline & Timed breeding & 11 & 3830 & 609 & 80 & 89 \\
\hline & Mixed & 36 & 9536 & 1046 & 83 & 278 \\
\hline Complete data & & 64 & 16,379 & 1171 & 86 & 481 \\
\hline
\end{tabular}

\footnotetext{
${ }^{1}$ Reproductive traits: DFB = days to first breeding; DO = days open; PR120 = pregnancy rate at $120 \mathrm{~d}$.

${ }^{2}$ Actual sire by herd management interaction levels accounted for $66 \%$ of the total possible sire by herd management subclasses for each trait.
}

results, and standard deviations among iterations were often smaller for sire models. For DFB, 2 subsets of data were analyzed with the base model: synchronized first breeding and nonsynchronized first breeding. Similarly for DO, 3 subsets were analyzed with the base model: synchronized first breeding, synchronized later breeding, and nonsynchronized. For both DFB and DO, 3 subsets based on herd management categories were analyzed with the expanded model. Burn-in levels were 10,000 followed by 25,000 iterations for parameter estimation. Parameter estimates were derived from the posterior distribution of the sample iterations. Means of the posterior distribution were reported for DFB and PR120, and medians were reported for DO. Heritabilities were estimated for each sample iteration.

Due to the discrete nature of PR120, a threshold analysis was implemented. Threshold models assure unobserved latent variables (or liability of pregnancy) with conceptual thresholds of success (Falconer and Mackay, 1996). If the liability exceeds the threshold, a pregnancy occurs. Because a threshold is not definable in a binary response situation, the threshold was assumed to be 0 , and this was the origin of the liability scale. The PROBIT.F90 software was used to estimate parameters in a sire model (Y. M. Chang, Department of Dairy Science, University of Wisconsin, Madison). The same data subsets and fixed effects were implemented as described for the DO analysis. Residual variance in the threshold sire model was set at 1.0, with a normal distribution of the latent variable. Bounded uniform priors were assumed for fixed effects and a conjugated prior was assumed for random sire variance. Burn-in length consisted of 20,000 iterations, and the median of 80,000 additional iterations was used to estimate variance parameters.

\section{RESULTS AND DISCUSSION}

\section{Effect of Synchronization on Reproductive Performance}

Means and standard deviations for DFB, by cow treatment and herd management subclasses, are in Table 3. Timed breeding herds attained first insemination an average of $5 \mathrm{~d}$ earlier than mixed herds and $12 \mathrm{~d}$ earlier than observed heat herds. Overall, mean DFB were slightly lower than previously reported (Hayes et al., 1992; Silva et al., 1992; Grosshans et al., 1997; Veerkamp et al., 2001). Differences in DFB may reflect differences in voluntary waiting periods between herds. In spite of timed breeding herds having the lowest mean DFB, synchronized cows across all categories of herd management had higher average DFB than nonsynchronized cows. In observed heat and mixed management herds, synchronization is clearly used selectively on cows with delayed or unobserved estrus. Even in timed breeding herds, cows that exhibit estrus before the targeted time of synchronization are bred on observed estrus. Thus, DFB is considerably less variable in timed breeding herds and for individual cows with synchronized first inseminations.

Median DO and mean PR120 by cow treatment and herd management subclasses are in Table 4. Median DO for the combined data set was similar to previous reports (Hayes et al., 1992; Silva et al., 1992; Marti and 
Table 3. Frequency distribution and mean and standard deviation of days to first breeding (DFB) by subclasses based on cow treatment and herd management.

\begin{tabular}{llccr}
\hline \multirow{2}{*}{$\begin{array}{l}\text { Herd } \\
\text { management } \\
\text { group }\end{array}$} & & \multicolumn{2}{c}{$\begin{array}{c}\text { Cow synchronization } \\
\text { treatment }^{1}\end{array}$} & Total \\
\cline { 2 - 4 } Observed heat & Category & First & None & 3465 \\
& No. of records & 545 & 2920 & 18.9 \\
& Percentage of records & 3.0 & 15.9 & 78.9 \\
& Mean DFB & 87.2 & 77.3 & 33.9 \\
Timed breeding & Standard deviation DFB & 30.0 & 34.7 & 4,129 \\
& No. of records & 2191 & 1938 & 22.5 \\
& Percentage of records & 11.9 & 10.6 & 67.1 \\
& Mean DFB & 73.3 & 60.2 & 14.3 \\
Mixed & Standard deviation DFB & 12.7 & 12.7 & 10,765 \\
& No. of records & 3699 & 7066 & 58.6 \\
& Percentage of records & 20.1 & 38.5 & 71.8 \\
Total & Mean DFB & 77.5 & 68.9 & 22.1 \\
& Standard deviation DFB & 17.7 & 23.6 & 18,359 \\
& No. of records & 6435 & 11,924 & 100.0 \\
& Percentage of records & 35.0 & 65.0 & 72.1 \\
\hline 1 & Mean DFB & 76.9 & 69.5 & 23.8 \\
\hline
\end{tabular}

${ }^{1}$ Cow synchronization treatment was defined as First (records with a synchronized first breeding) and None (records with nonsynchronized first breeding).

${ }^{2}$ Mixed herds had nearly equal proportions of synchronized and nonsynchronized breeding records.

Funk, 1994; Grosshans et al., 1997; Dematawewa and Berger, 1998; Abdallah and McDaniel, 2000). Records with no synchronization had lower DO and higher PR120 than records synchronized either before or after first insemination. Records synchronized after first breeding had a substantially higher DO and lower
PR120 than any other data set. A likely explanation for these results is that producers use synchronization selectively on animals that have not shown estrus by a given time. Also, poor heat detection around $21 \mathrm{~d}$ after breeding may increase DO. Synchronized breeding events for problem animals are a more likely cause for

Table 4. Frequency distribution, median, and standard deviation of days open (DO), and mean pregnancy rate at $120 \mathrm{~d}$ (PR120) by subclasses based on cow treatment and herd management.

\begin{tabular}{llcccc}
\hline \multirow{2}{*}{$\begin{array}{l}\text { Herd } \\
\text { management } \\
\text { group }\end{array}$} & & \multicolumn{2}{c}{ Cow synchronization treatment ${ }^{1}$} & \\
\cline { 2 - 4 } Observed heat & Category & First & Later & None & Total \\
& No. of records & 439 & 270 & 2,304 & 3013 \\
& Percentage of records & 2.7 & 1.6 & 14.1 & 18.4 \\
& Median DO & 140 & 242 & 141 & 144 \\
& Standard deviation DO & 93 & 100 & 101 & 102 \\
Timed breeding & Mean PR120, & 37.6 & 6.7 & 40.8 & 37.3 \\
& No. of records & 1969 & 711 & 1150 & 3830 \\
& Percentage of records & 12.0 & 4.3 & 7.0 & 23.4 \\
& Median DO & 125 & 211 & 89 & 127 \\
Mixed & Standard deviation DO & 93 & 101 & 71 & 97 \\
& Mean PR120, $\%$ & 49.5 & 20.8 & 48.0 & 48.5 \\
& No. of records & 3132 & 1617 & 4787 & 9536 \\
& Percentage of records & 19.1 & 9.9 & 29.2 & 58.2 \\
Total & Median DO & 152 & 211 & 117 & 146 \\
& Standard deviation DO & 93 & 99 & 97 & 101 \\
& Mean PR120, \% & 33.2 & 15.1 & 50.3 & 38.7 \\
& No. of records & 5540 & 2598 & 8241 & 16,379 \\
& Percentage of records & 33.8 & 15.8 & 50.3 & 100.0 \\
& Median DO & 142 & 213 & 119 & 142 \\
& Standard deviation DO & 93 & 100 & 96 & 100 \\
& Mean PR120, \% & 39.3 & 15.8 & 49.6 & 40.7 \\
\hline
\end{tabular}

${ }^{1}$ Cow synchronization treatment was defined as First (records with a synchronized first breeding), Later (records with nonsynchronized first breeding but synchronized subsequent breedings), and None (records with nonsynchronized breedings).

${ }^{2}$ Mixed herds had nearly equal proportions of synchronized and nonsynchronized breeding records. 
Table 5. Variance and heritability estimates with sire models for days to first breeding. Standard deviations (SD) among iterations are in parentheses.

\begin{tabular}{lllll}
\hline Approach & Data subset & $\begin{array}{l}\text { Sire } \\
\text { variance } \\
\text { (SD) }\end{array}$ & $\begin{array}{l}\text { Residual } \\
\text { variance } \\
\text { (SD) }\end{array}$ & $\begin{array}{l}\text { Estimated } \\
\text { heritability } \\
\text { (SD) }\end{array}$ \\
\hline Cow synchronization treatment & First & $2.5(1.0)$ & $168(3)$ & $0.06(0.02)$ \\
Herd management group & None & $1.6(1.1)$ & $483(6)$ & $0.02(0.01)$ \\
& Observed heat & $18.6(10.2)$ & $893(23)$ & $0.09(0.04)$ \\
& Timed breeding & $1.2(1.1)$ & $122(3)$ & $0.05(0.03)$ \\
Complete data & Mixed & $1.0(0.7)$ & $337(5)$ & $0.01(0.01)$ \\
& Base model & $2.7(1.1)$ & $432(5)$ & $0.03(0.01)$ \\
& Expanded model & $1.6(0.8)$ & $397(4)$ & $0.02(0.01)$ \\
& Interaction model ${ }^{1}$ & $0.5(0.5)$ & $394(4)$ & $0.01(0.01)$ \\
\hline
\end{tabular}

${ }^{1}$ Sire by herd management interaction variance was 5.0 (1.6).

higher DO in later-synchronized records than nonsynchronized records. Most later-synchronized records were in mixed management herds. This suggests that mixed management herds were primarily using synchronization to aid problem cows not pregnant by a particular time. The rationale behind separating data based on first-synchronized records and later-synchronized records was to differentiate between synchronization for first breeding and synchronization used to treat problem cows after the first breeding. Median DO and mean PR120 varied moderately among herd management classifications. Timed breeding herds had lower DO by nearly one estrous cycle and 10\% higher PR120 than either observed heat herds or mixed management herds.

Mean DFB and median DO were lower for herds strictly applying timed breeding but were not lower for synchronized breedings in general. These results suggest that synchronization protocols may be selectively applied in certain herds, while a more uniform application occurs in other herds. The distinct reproductive environments are a result of producer choice of synchronization protocol. These choices may be based on the type of protocol and the level of implementation of the protocols. Some producers do not implement any synchronization and rely strictly on standing heats, but others apply synchronization to all cows after a certain voluntary waiting period. Synchronization may be used to treat problem cows that have not been bred or conceived after a certain number of days postpartum. This selective use of synchronization complicates genetic analysis and interpretation of results, because sire daughter groups may differ in frequency and time in which synchronization is used.

\section{Genetic Parameter Estimates}

Genetic parameter estimates for DFB, DO, and PR120 are shown in Tables 5, 6, and 7, respectively. Estimates are reported from the base, expanded, and interaction models for the complete data set. Results are also shown for subsets based on cow treatment and herd management categories. The software did not provide standard errors of parameter estimates, so we report the standard deviations among iterations to characterize stability of the estimates. In general, and as expected, these standard deviations were smaller for the complete data set than the subsets. In many cases, the standard deviations were substantially larger for the subset of herds managed by observed heat than for other subsets. These large standard deviations tend to be associated with substantially larger parameter estimates. Results for DFB and DO for the observed heat subset are less credible.

The simplest way to account for potential synchronization influences in genetic evaluations is to include cow synchronization treatment as a fixed effect. In the complete data set, residual variances were reduced by about $8 \%$ for DFB and $11 \%$ for DO in the expanded model, compared with the base model. Sire variances were smaller for the expanded model than the base model for all traits, suggesting confounding between sire and cow synchronization treatments. However, the amount of reduction was not consistent and ranged from as little as $3 \%$ for sire variances in PR120 to as much as $40 \%$ for sire variances in DFB. Sire variances for DO in the expanded model were reduced by around $30 \%$. Heritability estimates were similar between the base, expanded, and interaction models, despite differences in variance estimates.

Including sire by herd management interaction in the model further reduced sire variances compared with the expanded model but had a negligible effect on residual variance. Sire variances were reduced by around $12 \%$ for DO and PR120. For DFB, sire variance was reduced by nearly $70 \%$. Although sire by herd management interaction accounted for a small portion of the total variance, especially for DO, its magnitude was as large or larger than sire variances for DFB and PR120. Estimation of both the interaction and genetic vari- 
Table 6. Variance and heritability estimates with sire models for days open. Standard deviations (SD) among iterations are in parentheses.

\begin{tabular}{lllll}
\hline & Data subset & $\begin{array}{l}\text { Sire } \\
\text { variance } \\
\text { (SD) }\end{array}$ & $\begin{array}{l}\text { Residual } \\
\text { variance } \\
\text { (SD) }\end{array}$ & $\begin{array}{l}\text { Estimated } \\
\text { heritability } \\
\text { (SD) }\end{array}$ \\
\hline Cow synchronization treatment & First & $61.3(24.7)$ & $6491(130)$ & $0.04(0.02)$ \\
& Later & $103.6(50.0)$ & $6369(196)$ & $0.06(0.03)$ \\
& None & $100.7(39.7)$ & $6308(105)$ & $0.06(0.02)$ \\
Herd management group & Observed heat & $57.5(55.9)$ & $6720(183)$ & $0.04(0.03)$ \\
& Timed breeding & $44.8(39.5)$ & $5908(140)$ & $0.03(0.03)$ \\
Complete data & Mixed & $47.9(27.3)$ & $6868(103)$ & $0.03(0.02)$ \\
& Base model & $80.7(25.1)$ & $7446(85)$ & $0.04(0.01)$ \\
& Expanded model & $54.2(20.7)$ & $6638(76)$ & $0.03(0.01)$ \\
& Interaction model ${ }^{1}$ & $46.4(19.3)$ & $6633(76)$ & $0.03(0.01)$ \\
\hline
\end{tabular}

${ }^{1}$ Sire by herd management interaction variance was 12.6 (14.1) model.

ances in these data may have been somewhat unreliable, due to confounding between sires and herd management groups. Minimum number of daughters per sire was 4 , and average progeny group size was 15 , so not all progeny groups were represented in all herd management categories.

Days to first breeding. Differences in variances were observed for DFB within cow treatment and herd management category. The base model was used to estimate parameters for data subsets based on cow treatment, whereas the expanded model was used for subsets based on herd management categories. Sire variance for DFB varied around $2 \mathrm{~d}^{2}$. Nonsynchronized records had $40 \%$ lower sire variances than synchronized records. Data subsets based on herd management category tended to have $10 \%$ lower sire variance than combined data. Sire variances were larger in observed heat herds than in any other data set. These larger variances were associated with large standard deviations among iterations, suggesting that these estimates were inflated.

Residual variances for DFB varied around $400 \mathrm{~d}^{2}$ in the complete data. Residual variances for synchronized cow treatments were only one-third of those in the com- plete data set and in nonsynchronized records and were lower in timed breeding herds than in other herd management categories. Residual variances were more heavily affected by synchronization than were sire variances or heritabilities. The variation across data sets in residual variances and sire variances caused heritability estimates to vary only moderately. Heritabilities for DFB ranged from 0.01 to 0.09 , with an average of 0.04 that was similar to heritabilities previously reported for DFB (Hayes et al.; 1992; Silva et al., 1992; Grosshans et al., 1997; Veerkamp et al., 2001). Standard deviations among iterations for heritabilities suggest that little real difference exists between cow treatment and herd management categories.

Days open. Sire variance for DO varied around 50 $\mathrm{d}^{2}$. Sire variances for DO tended to be at least $25 \%$ higher within cow treatment categories than in the combined data set. Sire variances were lower for synchronized first inseminations than records with breedings that were nonsynchronized or synchronized after the first insemination. Data subsets based on herd management category tended to have $10 \%$ lower sire variance than the combined data. Sire variances were slightly larger in observed heat herds than in any other data

Table 7. Variance and heritability estimates for sire model for pregnancy rate at $120 \mathrm{~d}$. Standard deviations (SD) among iterations are in parentheses.

\begin{tabular}{llll}
\hline & Data subset & $\begin{array}{l}\text { Sire } \\
\text { variance } \\
\text { (SD) }\end{array}$ & $\begin{array}{l}\text { Estimated } \\
\text { heritability } \\
\text { (SD) }\end{array}$ \\
\hline Cow synchronization treatment & First & $0.043(0.017)$ & $0.16(0.06)$ \\
& Later & $0.058(0.046)$ & $0.22(0.15)$ \\
Herd management group & None & $0.057(0.015)$ & $0.22(0.05)$ \\
& Observed heat & $0.041(0.019)$ & $0.16(0.07)$ \\
Complete data set & Timed breeding & $0.071(0.025)$ & $0.26(0.09)$ \\
& Mixed & $0.036(0.010)$ & $0.14(0.04)$ \\
& Base model & $0.029(0.007)$ & $0.11(0.03)$ \\
& Expanded model & $0.028(0.007)$ & $0.11(0.03)$ \\
& Interaction model ${ }^{1}$ & $0.025(0.007)$ & $0.10(0.03)$ \\
\hline
\end{tabular}

${ }^{1}$ Sire by herd management interaction variance was $0.021(0.006)$. 
set and were associated with large standard deviations among iterations, suggesting that these estimates are inflated.

Residual variances varied around $6500 \mathrm{~d}^{2}$ for DO in the complete data. They deviated by only about $3 \%$ among cow synchronization treatment categories and $14 \%$ among herd management categories. Nonsynchronized records tended to have slightly lower residual variances than synchronized records. Residual variances were lower in timed breeding herds than other herd management categories. Heritability estimates for DO ranged from 0.03 to 0.07 , with an average of 0.04 that was similar to those previously reported (Hayes et al., 1992; Silva et al., 1992; Marti and Funk, 1994; Grosshans et al., 1997; Dematawewa and Berger, 1998; Abdallah and McDaniel, 2000). Standard deviations among iterations for heritabilities suggest little real difference exists between cow treatment and herd management categories.

Pregnancy rate at 120 d. Sire variances for PR120 tended to be at almost $50 \%$ higher within cow treatment categories and $15 \%$ higher in herd management categories, when compared with the complete data. Sire variances were higher in timed breeding herds than either observed heat or mixed herds and the larger sire variances were associated with larger standard deviation among iterations. Heritability for PR120 was higher and varied more among data subsets than with the complete data, and estimates were higher than either DFB or DO, with an average of 0.15 .

\section{Evaluation of Reproductive Traits and Data Collection}

Under conventional artificial insemination and estrus detection, DFB measures various aspects of reproductive function. When producers use synchronization to manage $\mathrm{DFB}$, the trait no longer measures postpartum reproductive function. In practice, this study found that all herds, even timed breeding herds, used synchronization protocols selectively; cows that exhibited estrus early in lactation were inseminated to observed heat. Herds varied widely in the stage of lactation at which synchronization was applied. Overall, synchronization diminished the validity of DFB as a measure of reproductive function.

An advantage for DO is that it measures the service period as well as the period represented by DFB. However, DO does not directly account for number of inseminations, and it is censored for cows that fail to conceive. Accurately determining the time of censoring may not be possible. To avoid the problem of censoring, PR120 is determined at an earlier stage of lactation but late enough that a high percentage of cows have at least one insemination. This investigation found higher heritabilities for PR120 than either DFB or DO. However, due to its binomial nature and lack of comparable research, further investigation into PR120 or similar traits with larger data sets is warranted.

Data for this project were collected from herds using Dairy Comp 305 that participated in a progeny testing program. The Dairy Comp 305 breeding codes associated with an insemination were used to determine synchronization treatment. Two limitations existed for this approach: the large number of noncoded breedings in the bred code records (a major reason for excluding herds early in the data collection process), and the variation in bred codes among herds. Some herds recorded specific synchronization protocols, whereas others reported only observed heat and timed breeding. Other limitations included the necessity for manual standardization of codes across herds and discontinuity in breeding information reported by individual herds. More uniform reporting of synchronization events would improve investigations into the effect of synchronization on genetic parameters.

\section{CONCLUSIONS}

With the increasing use of protocols to synchronize estrus or ovulation in management of reproduction, it is important to understand its effects on genetic parameters of reproductive traits. Average heritabilities for complete data were 0.03 for $\mathrm{DFB}, 0.04$ for $\mathrm{DO}$, and 0.11 for PR120. Synchronization substantially reduced residual variances for all traits and moderately reduced sire variances. No effect of synchronization was seen for heritability estimates of the 3 traits. Including a fixed effect for cow synchronization treatment and a random effect for sire by herd management interaction also reduced variance estimates, with a slight reduction in heritability. Due to small progeny group sizes and the existence of sire by herd management combinations with no records, further investigation of sire by herd management interaction should occur before it is included in genetic models. Future studies should consider the fact that not all synchronization protocols permit timed inseminations; some still require estrus detection before insemination. Results indicated that heterogeneous residual variance resulted from synchronization treatments. Separate genetic evaluations by treatment category are not recommended, but methods to adjust for heterogeneous variance should be applied.

\section{ACKNOWLEDGMENTS}

The authors thank R. D. Welper, Alta Genetics, for providing the herd data utilized in this investigation. 
Statistical consultation and computer program guidance was supported by Yu-Mei Chang.

\section{REFERENCES}

Abdallah, J. M., and B. T. McDaniel. 2000. Genetic parameters and trends of milk, fat, days open, and body weight after calving in North Carolina experimental herds. J. Dairy Sci. 83:1364-1370.

Cartmill, J. A., S. Z. El-Zarkouny, B. A. Hensley, T. G. Rozell, J. F. Smith, and J. S. Stevenson. 2001. An alternative AI breeding protocol for dairy cows exposed to elevated ambient temperatures before or after calving or both. J. Dairy Sci. 84:799-806.

Dematawewa, C. M. B., and P. J. Berger. 1998. Genetic and phenotypic parameters for 305-day yield, fertility, and survival in Holsteins. J. Dairy Sci. 81:2700-2709.

Falconer, D. S., and T. F. C. Mackay. 1996. Pages 299-300 in Introduction to Quantitative Genetics. 4th ed. Longman Group Ltd., Essex, UK.

Fricke, P. M. 2002a. Reproduction practices to extend cow longevity. Online. Available http://www.wisc.edu/dysci/uwex/rep_phys/ pubs/longevity502.pdf. Accessed Aug. 3, 2002.

Fricke, P. M. 2002b. Scanning the future-Ultrasonography as a reproductive management tool for dairy cattle. J. Dairy Sci. 85:1918-1926.

Grosshans, T., Z. Z. Xu, L. J. Burton, D. L. Johnson, and K. L. Macmillan. 1997. Performance and genetic parameters for fertility of seasonal dairy cows in New Zealand. Livest. Prod. Sci. 51:41-51.

Hageman, W. H., II, G. E. Shook, and W. J. Tyler. 1991. Reproductive performance in genetic lines selected for high or average milk yield. J. Dairy Sci. 74:4366-4376.
Hayes, J. F., R. I. Cue, and H. G. Monardes. 1992. Estimates of repeatability of reproductive measures in Canadian Holsteins. J. Dairy Sci. 75:1701-1706.

Jobst, S. M., R. L. Nebel, M. L. McGilliard, and K. D. Peizer. 2000 Evaluation of reproductive performance in lactating dairy cows with prostaglandin $\mathrm{F}_{2 \alpha}$, gonadotropin-releasing hormone, and timed artificial insemination. J. Dairy Sci. 83:2366-2372.

Marti, C. F., and D. A. Funk. 1994. Relationship between production and days open at different levels of herd production. J. Dairy Sci. 77:1682-1690.

Pursley, J. R., M. R. Kosorok, and M. C. Wiltbank. 1997. Reproductive management of lactating dairy cows using synchronization of ovulation. J. Dairy Sci. 80:301-306.

Pursley, J. R., M. O. Mee, and M. C. Wiltbank. 1995. Synchronization of ovulation in dairy cows using $\mathrm{PGF}_{2 \alpha}$ and $\mathrm{GnRH}$. Theriogenology 44:915-923.

Royal, M. D., A. P. F. Flint, and J. A. Woolliams. 2002. Genetic and phenotypic relationships among endocrine and traditional fertility traits and production traits in Holstein-Friesian dairy cows. J. Dairy Sci. 85:958-967.

Silva, H. M., C. J. Wilcox, W. W. Thatcher, R. B. Becker, and D. Morse. 1992. Factors affecting days open, gestation length, and calving interval in Florida dairy cattle. J. Dairy Sci. 75:288-293.

Van Arendonk, J. A. M., R. Hovenier, and W. De Boer. 1989. Phenotypic and genetic association between fertility and production in dairy cows. Livest. Prod. Sci. 21:1-12.

Veerkamp, R. F., E. P. Koenen, and G. De Jong. 2001. Genetic correlations among body condition score, yield, and fertility in firstparity cows estimated by random regression models. J. Dairy Sci. 84:2327-2335. 